\title{
APLICACIÓN DEL QFD A LA INDUSTRIA REFRESQUERA DE SAN LUIS POTOSÍ, MÉXICO
}

\author{
Juan Manuel Izar Landeta*, Carmen Berenice Ynzunza Cortés**
}

\begin{abstract}
Izar-Landeta J.M., Ynzunza-Cortés C.B. Aplicación del QDF a la industria refresquera de San Luis Potosí, México. Hitos de Ciencias Económico Administrativas 2013;19 (53): 7-16.
\end{abstract}

\section{RESUMEN}

Objetivo: Mostrar cómo el despliegue de funciones de calidad (QFD), herramienta para la planeación y solución de problemas, puede ser utilizada en la industria refresquera para explorar los requerimientos técnicos y el nivel de satisfacción de los clientes con los atributos ofrecidos en el producto.

Material y Método: El enfoque propuesto de la metodología del QFD, utiliza la casa de la calidad, en la cual se considera no sólo los aspectos inherentes a las relaciones entre los requerimientos del cliente y las características del producto, sino también las correlaciones entre las características de éstos y sus procesos. Para demostrar la utilidad del QFD, se toman como referencia 4 marcas de refrescos ofertadas en el Estado de San Luis Potosí. En el estudio los clientes dan su opinión sobre el producto analizado, respondiendo a una encuesta, y del mismo modo, los fabricantes de refrescos expresan su opinión a través de una entrevista personal.

Resultados: Los resultados alcanzados permiten determinar las características técnicas más importantes para el cliente, así como de los procesos del embotellado de refrescos para garantizar la satisfacción de sus clientes, mostrando así la utilidad que la aplicación del QFD tiene como herramienta para la mejora continua y dar un enfoque de orientación al cliente.
Izar-Landeta J.M., Ynzunza-Cortés C.B. Application of QFD (Quality Function Deployment) to the soft drink industry in San Luis Potosi, Mexico. Hitos de Ciencias Económico Administrativas 2013;19 (53): 7-16.

\section{ABSTRACT}

Objective: This research aims to show how the quality function deployment (QFD), tool for planning and problem solving, can be used in the soft drink industry to explore the technical requirements and level of satisfaction of consumers with the attributes offered by product.

Material and Method: The approach proposed by the QFD methodology, uses the house of quality, which considers not only the aspects inherent in the relationship between customer requirements and product characteristics, but also the correlations between their characteristics and their processes. To demonstrate the utility of QFD, 4 soft drink brands that are offered in the state of San Luis Potosi are considered as reference. In the study, the customers give their own opinion regarding the analyzed product by answering a survey, and similarly, the soft drink manufacturers express their opinion through a personal interview.

Results: The given results in this study let to determine the most important technical soft drink characteristics for the client, as well as the soft drink bottling processes to guarantee customer satisfaction, showing in this way, the usefulness of applying the methodology of QFD as a tool for the product continuous improvement and customer orientation approach.
Palabras clave: QFD. Requerimientos técnicos. Calidad. Despliegue de funciones. Satisfacción del cliente.
Key words: QFD. Technical Requirements. Quality. Function Deployment. Customer satisfaction.

DIRECCIÓN PARA RECIBIR CORRESPONDENCIA: Correo electrónico: jmizar@uaslp.mx

*Doctor en Administración. Profesor-Investigador. Universidad Autónoma de San Luis Potosí.

** Doctor en Administración. Profesora-Investigadora de la Universidad Tecnológica de Querétaro. 
I incremento de la competencia, ha forzado a las organizaciones a transformar sus procesos productivos y a diseñar nuevos productos y servicios para satisfacer las necesidades y demandas de sus clientes. Los clientes actuales son más demandantes y conscientes de la calidad; y día a día están ganando más importancia, por lo que su voz necesita ser cuidadosamente traducida a requerimientos técnicos. Alinear los productos y servicios a estas necesidades es todo un desafío. El despliegue de funciones de calidad (QFD) es una técnica diseñada originalmente para este propósito; es una manera de hacer que la voz del cliente sea escuchada. Un proceso sistemático para capturar los requerimientos del cliente y traducirlos en acciones que deben realizarse a través de la cadena de valor (Shil y Das, 2008). El resultado es un nuevo conjunto de objetivos a alcanzar para los diseñadores, la gente de producción e incluso los proveedores, buscando satisfacer los deseos del cliente.

Este enfoque de orientación al cliente es la columna vertebral para el desarrollo de nuevos productos, considerando por supuesto las necesidades del cliente. Básicamente, en el QFD las necesidades del cliente se traducen en atributos de diseño, que posteriormente se trasladan a los procesos productivos y requerimientos de calidad. Los clientes satisfechos son la clave para el éxito competitivo; sin embargo, el cómo incorporar las necesidades presentes y futuras del cliente, solicitadas o no por él, en productos y servicios de una compañía, no es tarea fácil. Muchos investigadores han encontrado la respuesta en el QFD, como una herramienta que permite identificar diferentes tipos de necesidades y niveles de satisfacción, esto por supuesto, en función de los requerimientos del cliente que se han satisfecho.

El cliente expresa en sus propias palabras sus necesidades, las cuales pueden ser capturadas a través de cuestionarios, observaciones y de muchas otras maneras. Sólo a través de la información recabada, será posible identificar el grado de satisfacción del cliente con el producto o servicio proporcionado. La casa de la calidad es la herramienta más utilizada en la metodología QFD, traduce la voz del cliente en especificaciones de los productos (Akao, 1990).
Con base en este análisis, el artículo se enfoca en la aplicación del QFD a la industria refresquera a través del diseño de la casa de calidad. El objetivo es identificar los requerimientos del cliente para este sector y conocer su nivel de satisfacción con los productos proporcionados. Se busca con ello comprender cómo a través del uso del QFD y otras herramientas, un proveedor de servicios o productos puede mejorarlos y alcanzar mayores niveles de satisfacción. La casa de calidad permite además cuantificar la importancia que atribuye el cliente a las características del producto o servicio y clasificarlos para que sean satisfechos. La razón detrás de la selección del QFD, es que es una técnica que ha sido utilizada ampliamente para mejorar el diseño de productos, la toma de decisiones, la satisfacción del cliente y permitir priorizar las medidas de desempeño (Carnevally y Miguel, 2008; Bottani, 2009). Por lo que en este trabajo se desarrolla la metodología para mostrar cómo el QFD puede ser utilizado y para enfatizar sus beneficios.

\section{Marco contextual}

La industria del embotellado de refrescos tiene más de 100 años de presencia en México y está representada por laAsociación Nacional de Productores de Refrescos y Aguas Carbonatadas, A. C. (ANPRAC), que fue fundada en el año 1945.

LaANPRAC agrupa a la mayoría de los embotelladores de refrescos y aguas carbonatadas del país. Al año 2008, esta industria contaba con 230 plantas embotelladoras distribuidas en todo el territorio nacional y una flota vehicular de más de 35,000 unidades, con un consumo de casi 1 millón 900 mil toneladas de azúcar y fructosa, lo que representa una demanda muy importante para los sectores agrícola y agroindustrial, que integran la cadena productiva de agricultores, cañeros y maiceros, y los obreros e industriales del azúcar y fructosa (ANPRAC, 2008).

El volumen de ventas de la industria de bebidas preparadas no alcohólicas en 2011, fue mayor a los 25 mil millones de litros, de los cuales $74 \%$ son refrescos, esta cifra representa un incremento de $9 \%$, muy superior al promedio de la industria, lo cual según la Organización de Naciones Unidas (ONU) se debió al Tratado de Libre Comercio de Norteamérica (TLC). En 2011, cada mexicano gastó un promedio de mil pesos en la compra de refrescos, cifra que representa 
entre 7 y $10 \%$ del ingreso de las familias mexicanas (Milenio, 2012).

Existe en el país cerca de 1 millón 400 mil puntos de venta de refrescos; de ellos, $75 \%$ son pequeñas empresas familiares que venden el refresco al público consumidor, $24 \%$ se vende en restaurantes, centros deportivos, hoteles y discotecas y el $1 \%$ restante, en tiendas de autoservicio (Organización Editorial Mexicana, 2008).

El Producto Interno Bruto de la industria embotelladora, representó el $8.7 \%$ del PIB de la División Alimentos Bebidas y Tabaco, $2.6 \%$ de la Industria Manufacturera y $0.4 \%$ del Producto Interno Bruto (PIB) Total, con un monto aproximado de 17 mil millones de dólares americanos (ANPRAC, 2008). Con una generación de 90,000 empleos directos y más de 700,000 en empleos indirectos, a través de su cadena productiva y en el conjunto de la actividad económica del país (24 Horas, 2012).

El consumo de refrescos está profundamente arraigado en los patrones de consumo de la población mexicana. Un estudio del Centro Rudd para Políticas Alimentarias y Obesidad de la Universidad de Yale, señala que México tiene un consumo per cápita anual de 163 litros de refresco, cifra superior en $40 \%$ al de los estadounidenses, lo que lo ubica en el primer lugar mundial (Excélsior, 2011). Por lo que algunas empresas han encontrado en México un mercado potencial para este tipo de productos, teniendo la industria refresquera, por supuesto, una participación significativa en la economía del país, aún y cuando su consumo se ha asociado a la aparición de varias enfermedades y se han tomado medidas para reducirlo.

Sin embargo, la industria refresquera ha tenido que acoplarse a las nuevas condiciones de su entorno, con cambios en la presentación, envase, o el tipo de endulzante, debido al incremento de los precios, aumento de la competencia, a la mayor preocupación por el cuidado de la salud, los altos costos de inversión en equipos enfriadores y despachadores, la demanda de nuevos canales de distribución, la especialización de marcas, las cargas impositivas, pero sobre todo a los nuevos patrones de consumo y requerimientos de sus clientes.

\section{Fundamentación teórica}

Akao y Mizuno desarrollaron el QFD en la década de los 60 , originalmente como un método para recopilar y analizar la voz del cliente, para desarrollar productos de alta calidad para satisfacer las necesidades del cliente.

Las funciones primarias del QFD eran el desarrollo de productos, la gestión de la calidad y el análisis de las necesidades del cliente; sin embargo, Chan y Wu (2002) encontraron que su uso se ha extendido a otros campos, como el diseño, la planeación, la toma de decisiones, la ingeniería, la administración y los costos. Ha sido utilizado ampliamente en muchas industrias en todo el mundo, alcanzando todas ellas beneficios significativos de su aplicación. Pullman y colaboradores (2002) señalan que el QFD puede ayudar a desarrollar soluciones únicas para las necesidades de los clientes, en virtud de que es una técnica que promueve el pensamiento creativo para el diseño de las características del producto que cumplan con las necesidades del cliente. Provee un medio para traducir las necesidades del cliente en los requerimientos de diseño apropiados para cada etapa del desarrollo del producto, por ejemplo estrategias de mercado, planeación, diseño e ingeniería de producto, evaluación de prototipos, desarrollo del proceso de producción, producción y ventas (Sullivan, 1986). Es una técnica bien conocida para la metodología de diseño de nuevos productos dirigidos al cliente, una herramienta para la planeación y solución de problemas, que ha pasado de la traducción de los requerimientos del cliente a los atributos técnicos de un producto ( Chen et al., 2004). EI QFD es una manera de hacer que la voz del cliente se escuche a través de la organización, un proceso sistemático de capturar los requerimientos del cliente y traducirlos en esos requerimientos que deberían ser cubiertos a través de la cadena de valor. El resultado es un nuevo conjunto de valores meta para los diseñadores, gente de producción y proveedores, para fabricar los productos deseados por los clientes (Shil y Das, 2008).

Nasser y Tabriz (2009) señalan que en años recientes, su uso se ha expandido notablemente para incluir la ingeniería simultánea, el benchmarking y un énfasis incrementado en temas relacionados con la estrategia de la organización, como el desarrollo de estrategias y planes de negocio que fortalezcan el desempeño financiero, operacional y relacionado con el cliente, sugieren su uso en la solución de problemas que 
permitan el acceso a la toma de decisiones, proveer medios de soporte, dar mayor énfasis a la gestión, así como a las habilidades técnicas, el conocimiento, acceso a futuros desarrollos y la gestión de la calidad. Chaudhuri y Bhattacharyyab (2009) sugieren que el QFD puede mejorar el desarrollo de nuevos productos, pero requiere que se tenga un buen proceso para capturar los requerimientos del cliente y traducirlos en información para ser utilizada por el QFD. Delice y Güngor (2011) consideran que una característica principal del QFD es determinar los valores meta para los requerimientos de diseño de un producto, con una visión para alcanzar un mayor nivel de satisfacción del cliente. Es un enfoque efectivo que permite a una empresa la implementación de la manufactura esbelta (Vinodh y Suresh, 2011).

Lee y colaboradores (2010) visualizan el QFD como un sistema de gestión de calidad comprensivo para considerar los requerimientos del cliente, comenzando cuidadosamente desde la conceptualización del producto, sin embargo, señalan que tiene sus limitaciones y que se han propuesto muchos modelos modificados. Uno de los enfoques recientes más populares es incorporar el proceso analítico de redes con el QFD.

Otros investigadores han utilizado el QFD con herramientas como la teoría difusa (fuzzy), o el modelo Kano, para tratar con los elementos imprecisos del proceso y como un enfoque novedoso para definir atributos que deberían ser incorporados o mejorados en un producto; identificar y analizar relaciones entre varios elementos, evaluar el estatus o implementación de un proceso, o detectar áreas en una organización que requieran ser mejoradas (Tontini, 2007; Chen y Huang, 2011; Chang y Chen, 2011).

\section{MATERIAL Y MÉTODO}

EI QFD es un medio de traducir los deseos y requerimientos del cliente en atributos técnicos apropiados en cada etapa del desarrollo y manufactura de un producto o servicio, considerando la voz del cliente, el análisis competitivo, la voz del equipo técnico, las correlaciones entre ambos, la comparación técnica respecto al desempeño del producto y los intercambios técnicos, utilizando una serie de matrices vinculadas, que son creadas con base en la evaluación de las demandas del usuario durante el proceso de desarrollo del producto o servicio. La matriz básica del QFD es conocida también como la casa de la calidad (Shil y Das, 2008).

La metodología del QFD tiene algunas variantes, según el autor que la utilice. En este apartado se explicará una de las más usuales encontradas en el ámbito de los negocios, la cual se sintetiza en lo que se denomina la casa de la calidad, que puede observarse en la figura 1 y consta de 6 etapas que son las siguientes (Izar, 2011): 1. Escuchar la voz del cliente. Consiste en captar lo que el cliente desea obtener al adquirir un producto o servicio, es decir sus requerimientos $(R C)$ y puede a su vez dividirse en varios pasos, como son identificar los clientes, identificar sus expectativas y la captación, manejo y clasificación de la información proveniente de los consumidores, esto significa el QUÉ del cliente.

2. Elaborar la matriz de planeación del producto en la parte derecha de la casa de la calidad. Lo que se hace es ubicar en la parte que muestra la figura 1 con el número 2 como Matriz de Planeación, las valoraciones que para cada RC han hecho los clientes del producto o servicio propio y el de la competencia, conforme a la información recabada en el paso anterior. A esta etapa también se le conoce como Benchmarking, ya que se comparan los resultados de los productos de un mismo sector, lo que proporciona información valiosa respecto a qué características del producto deben mejorarse, o bien si la empresa debe redefinir su segmentación de mercado.

\section{Establecer las características técnicas del producto.} A esta etapa algunos autores le llaman la voz de la compañía, pues consiste en elaborar un listado de las características técnicas (CT) que debe tener el producto o servicio para satisfacer los requerimientos del cliente (RC). Esta tarea normalmente la lleva a cabo el departamento de ingeniería de la organización y cada CT debe poder medirse mediante algún indicador. Cada CT debe satisfacer al menos un $\mathrm{RC}$, pues de no ser así, no tendría ningún sentido que apareciese, ya que se estarían considerando aspectos del producto que no tienen ningún valor para el cliente. En este paso debe evitarse la redundancia de CT; esto es el cómo para la compañía. 
4.Definir la matriz de relaciones entre los requerimientos del cliente y las características técnicas del producto. En esta matriz se ubican los $\mathrm{RC}$ como renglones y los $\mathrm{CT}$ como columnas y para cada celda se determina aunque sea subjetivamente, el grado de influencia o correlación entre el RC del renglón y el CT de la columna, habiendo 4 posibilidades: grado fuerte de correlación entre el RC y el CT, valorado con puntuación de 9; grado medio de correlación, que vale 3 puntos; correlación débil, evaluada con un punto; y que no exista correlación, en cuyo caso se evalúa con cero y dicha celda se deja en blanco. En esta etapa algunos autores señalan que una de las debilidades del QFD es que no considera correlaciones de tipo negativo entre un RC y un CT. Una vez evaluadas todas las celdas, lo que se hace es calcular para cada CT su puntuación, lo cual se hace mediante la sumatoria de los productos del grado de correlación antes dado, multiplicado por el grado de importancia del RC, según la información recabada en la primera etapa. La puntuación obtenida de cada CT es un reflejo de su importancia relativa para alcanzar la satisfacción del cliente.

5.Definir correlaciones entre las características técnicas del producto, las cuales se ubican en el techo de la casa de la calidad. Esta etapa se efectúa en el techo de la casa y simplemente consiste en evaluar para cada par de CT su grado de correlación existente, el cual puede ser positivo fuerte, positivo débil, negativo fuerte, negativo débil, o que no haya correlación. Las correlaciones positivas aplican cuando al implementar acciones para conseguir el primer CT del producto, se facilita que se obtenga el segundo y las negativas van en sentido opuesto. De esta etapa suelen surgir ideas que representan oportunidades para mejorar el producto o servicio y las correlaciones negativas resultantes representan objetivos en conflicto, los cuales deben resolverse por parte del departamento de ingeniería o diseño de la organización.

\section{Fijarmetas a alcanzaren cuanto a las características} del producto, que van en el fondo de la casa de la calidad. En esta etapa se fija un valor objetivo deseable expresado en unidades para cada CT del producto y se califica respecto a dicho valor meta tanto al artículo de la compañía, como a los de la competencia, de modo que esto representa benchmarking en cuanto a las características técnicas de los productos, lo cual puede generar ideas para la mejora (ver figura 1).

\section{FIGURA 1. CASA DE LA CALIDAD CON SUS 6 PARTES}

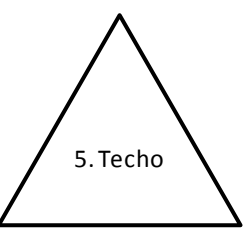

3. Características

técnicas del producto $(\mathrm{CT})$

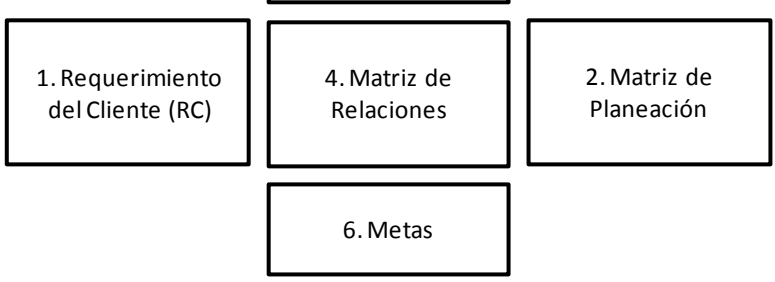

Fuente: Elaboración propia.

Una vez establecida la metodología del QFD, se determinó aplicar la misma al estudio de la industria refresquera en el estado de San Luis Potosí, para lo cual se hizo uso de datos primarios y secundarios, utilizando el QFD como herramienta de análisis, debido a que su uso ha sido ampliamente probado con éxito en diversos campos y provee información importante para garantizar la satisfacción del cliente. Por lo que el propósito de este trabajo es utilizar el QFD para identificar y cuantificar los requerimientos del cliente y el nivel de satisfacción del mismo con los atributos técnicos del producto.

El estudio inició con la realización de una encuesta a 100 clientes sobre los atributos deseables de un refresco (los RC del cliente), medidos en una escala Likert de 5 puntos, siendo 1 el menos importante hasta 5 el valor máximo; posteriormente se hizo una reunión con los responsables del área operativa de una de las 4 empresas comprendidas en el estudio, con el fin de determinar las características principales del producto, así como los procesos llevados a cabo para su producción y colocación en el punto de venta. Posteriormente, se procedió a la aplicación del QFD. 


\section{RESULTADOS}

Conforme al primer paso de la metodología del QFD, se determinaron mediante una encuesta los principales requerimientos del cliente $(R C)$ respecto a un refresco, que han sido 8 , los cuales se muestran en la tabla I, con su grado de importancia y agrupación ya incluido.

TABLA I. RC DEL CLIENTE PARA EL CASO DE UN REFRESCO

\begin{tabular}{clc}
\hline $\begin{array}{c}\text { RC } \\
\text { Nivel 1 }\end{array}$ & \multicolumn{1}{c}{$\begin{array}{c}\text { RC } \\
\text { Nivel 2 }\end{array}$} & $\begin{array}{c}\text { Grado de } \\
\text { Importancia }\end{array}$ \\
\hline \multirow{2}{*}{$\begin{array}{c}\text { Calidad del } \\
\text { Producto }\end{array}$} & Que tenga buen sabor & 5 \\
& Que contenga la & 5 \\
& cantidad correcta & 3 \\
Aspectos & Envase higiénico & \\
Visuales & Envase agradable & 4 \\
& Tapa en buen estado & 2 \\
\hline \multirow{2}{*}{ Mercadeo } & Buen precio & 1 \\
& Prestigio de la marca & 2 \\
\hline
\end{tabular}

Fuente: Elaboración propia.

TABLA II. MATRIZ DE PLANEACIÓN DE LOS 4 REFRESCOS

Nivel 1
1. Que tenga buen sabor

Fuente: Elaboración propia.
Puede observarse que se han clasificado $3 \mathrm{RC}$ de nivel 1: la calidad del producto que agrupa a $3 \mathrm{RC}$ de nivel 2: que el refresco tenga buen sabor, que esté frío y con la cantidad correcta; los aspectos visuales, donde hay también $3 \mathrm{RC}$ de nivel 2: la higiene del envase, su aspecto y que la tapa esté en buen estado; y los factores de mercadeo, donde han quedado $2 \mathrm{RC}$ de nivel 2: el precio del refresco y el prestigio de la marca.

En cuanto al grado de importancia, conforme a los resultados de las encuestas, los RC más importantes han sido que el refresco tenga buen sabor y esté frío y el de menor importancia es el aspecto de la tapa.

El segundo paso, consistió en elaborar la matriz de planeación, la información se obtiene también de las encuestas aplicadas a los clientes, donde califican para cada $\mathrm{RC}$ al producto de la compañía, denominado con la letra $D$, con otros 3 competidores, A, B y C, dicha matriz se muestra en la tabla II.

En la matriz se han dibujado los perfiles para cada marca, donde aquellas líneas que queden hacia el lado izquierdo indican las mejores marcas, tal es el caso de C (línea roja) y la marca propia D (línea azul), mientras que las peores marcas son la $A$ (línea verde) y la $B$ (línea celeste). En esta matriz se observa que la empresa etiquetada con la letra C parece ser la mejor marca, no obstante que es la del precio más alto. C supera a D en 5 de los RC, mientras que $\mathrm{D}$ lo hace sólo en 2.

Conforme al tercer paso, con la información proporcionada por el personal del área operativa, se determinaron las características principales del producto (CT), resultando 9 , que son: (1) contenido del refresco, (2) grados 
brix (es una medida del contenido de azúcar), (3) el coronado (buena tapa), (4) la limpieza del envase, (5) la calidad del envase, (6) el contenido de gas carbónico, (7) el precio, (8) la publicidad y (9) la labor de mercadeo del departamento de ventas.

Con estos datos, conforme al paso cuatro, se desarrolla la matriz de relaciones entre los $\mathrm{RC}$ ubicados como renglones y los CT situados como columnas, la cual se presenta en la tabla III.

Se ha evaluado con 9 una correlación fuerte, con 3 una mediana y con 1 una correlación débil y las celdas que se han dejado en blanco son aquellas donde no ha habido correlación entre el RC y el CT, así para el caso del tercer RC que es el contenido correcto del refresco, se ha correlacionado fuertemente con el primer $\mathrm{CT}$, que es precisamente el llenado del refresco.

Luego el valor de puntuación (renglón Pts) se calcula para cada CT como la sumatoria de los productos del grado de correlación multiplicado por el grado de importancia del $\mathrm{RC}$ respectivo, lo cual se ilustra para el caso del CT número 8 , cuya sumatoria es la siguiente:

$$
\text { Pts }(\text { CT } 8)=3 \times 4+9 \times 2+3 \times 1+9 \times 3=60
$$

La sumatoria de los 9 CT es 402 y el último renglón contiene la fracción porcentual de cada CT respecto a la puntuación total, lo que da una idea de la importancia relativa de cada CT. De esta manera, el CT más importante para lograr los RC deseados por

\section{FIGURA 2. CORRELACIÓN ENTRE LOS CT}

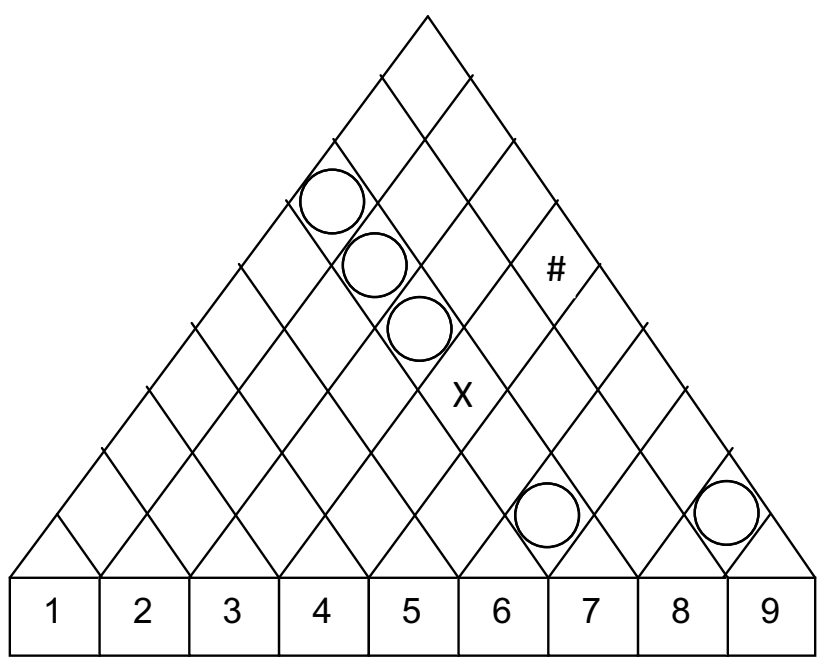

Tipo de Correlación:

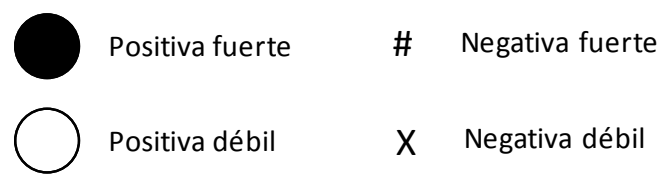

Fuente: Elaboración propia.

los clientes, es el noveno con 72 puntos, o un $17.9 \%$ y que justamente corresponde a la labor de mercadeo del refresco.

El quinto paso del QFD corresponde al grado de correlación entre los CT el cual se presenta en la figura 2 y corresponde al techo de la casa de calidad. En la figura puede verse que el séptimo CT (el precio) correlaciona positivo débil con el refresco con contenido correcto, los grados brix, la tapa y el contenido de gas. Por su parte, las únicas correlaciones negativas han resultado para el cuarto $\mathrm{CT}$, que es el envase sucio, que ha correlacionado débil con el precio y fuerte con el mercadeo, lo cual es una advertencia de los impactos negativos que puede tener una alta tasa de envase sucio.

Fuente: Elaboración propia. 
TABLA IV. VALORES META PARA LOS CT Y COMPARATIVO CON OTRAS MARCAS

\begin{tabular}{lccccccccc}
\hline \multicolumn{1}{c}{ CT } & 1 & 2 & 3 & 4 & 5 & 6 & 7 & 8 & 9 \\
\hline Meta & $0.08 \%$ & 10.8 & $0.05 \%$ & $0.1 \%$ & $0.28 \%$ & 2.9 & 6.50 & 9.5 & 9.8 \\
& & & & & & grs $/ \mathrm{u}$ & $\$ / \mathrm{u}$ & & \\
Mal & & & & & B & & & B & \\
Regular & B & A C & A & B & A D & A C & C & A D & A B D \\
Bien & A C D & B D & B C D & ACD & C & B D & A B D & C & C \\
\hline
\end{tabular}

Fuente: Elaboración propia.

En el sexto paso se definen las metas de cada CT, lo que se sintetiza en la tabla IV, donde aparece el valor objetivo para cada CT, que lo define el departamento operativo correspondiente, como puede ser el de producción para los primeros 6 CT y el de ventas y mercadeo para los últimos 3.

Este mismo procedimiento se siguió para los CT, clasificándolos en bien, regular o mal, según el criterio de los departamentos operativos de cada organización.

En la tabla puede verse que la marca propia (D) al igual que la $\mathrm{C}$, aparecen bien clasificadas en la mayoría de los CT. Asimismo en los valores meta se utilizan las unidades que correspondan a cada CT, siendo para la mayoría de estos fracciones porcentuales, excepto en el segundo CT (grados brix), donde el valor meta es 10.80, el sexto CT (contenido de gas) cuyo valor meta es 2.9 gramos por unidad, el séptimo CT (precio) y los dos últimos, donde se asigna una calificación entre cero y diez, que depende de la verificación que hace el departamento de supervisión en cuanto a la publicidad y mercadeo.

Finalmente en este ejercicio de dos fases, se colocan ahora los CT como renglones con sus puntuaciones porcentuales obtenidas en la casa 1 y los procesos realizados por las envasadoras como columnas para hacer una segunda casa. Para lo cual lo primero es identificar los procesos, los cuales son: (1) preparación del jarabe terminado, (2) lavado de envases, (3) transportación del envase en el proceso de embotellado, (4) inspección de envases, (5) mezclado de jarabe y gas carbónico, (6) llenado, (7) colocación de las tapas, (8) empacado del refresco y (9) puesta en el punto de venta en el mercado.

Luego se definen las correlaciones entre los CT y los procesos tal y el resultado se muestra en la tabla $\mathrm{V}$, con su puntuación y valor porcentual en los dos últimos

TABLA V. MATRIZ DE RELACIONES ENTRE LOS CT Y LOS PROCESOS

\begin{tabular}{ccccccccccc}
\hline $\begin{array}{c}\text { Proceso } \\
\text { CT }\end{array}$ & Pts. & 1 & 2 & 3 & 4 & 5 & 6 & 7 & 8 & 9 \\
\hline 1 & 6.7 & & & & & & 9 & & & \\
2 & 11.2 & 9 & & & & & & & & \\
3 & 13.4 & & & & & & & 9 & & \\
4 & 10.5 & & 9 & & 3 & & & & & 1 \\
5 & 7.5 & & 1 & 3 & & & & & 3 & 1 \\
6 & 11.2 & & & & & 9 & & & & \\
7 & 6.7 & & & & & & & & & \\
8 & 14.9 & & & & 3 & & & & & 1 \\
9 & 17.9 & & & & & & & & & 9 \\
Pts & 799.7 & 100.8 & 102 & 22.5 & 76.2 & 100.8 & 60.3 & 120.6 & 22.5 & 194 \\
$\%$ & 100.0 & 12.6 & 12.8 & 2.8 & 9.5 & 12.6 & 7.5 & 15.1 & 2.8 & 24.3 \\
\hline
\end{tabular}

Fuente: Elaboración propia. 
renglones. En la tabla se observa que el proceso más importante es el noveno, que es la puesta del producto en el punto de venta, seguida por el séptimo proceso, que es la colocación de las tapas, ya que un refresco mal tapado pierde gas y por ende su sabor.

\section{DISCUSIÓN}

Este trabajo prueba la utilidad que tiene la aplicación de la metodología del QFD, cuya filosofía es «escuchar la voz del cliente» en la identificación de las características clave de un producto para satisfacer los requerimientos del cliente. Es una técnica para la mejora continua, que contribuye a detectar nichos de oportunidad para los productos y/o servicios actuales o para el desarrollo de nuevos productos. Todas las empresas, tanto de manufactura como de servicios, deberían hacer uso de esta herramienta para mejorar sus procesos, productos y servicios, y con ello su rentabilidad.

Las características técnicas más importantes en el caso de los refrescos embotellados fueron las labores de mercadeo, la publicidad y el buen estado de las tapas del refresco, lo que refuerza la importancia de estas actividades para lograr la satisfacción del consumidor.

En cuanto a los procesos, los más importantes fueron la puesta del producto en el punto de venta, seguida de varios procesos que tienen que ver con la producción como son la colocación de las tapas, el lavado de los envases, la preparación del jarabe terminado y la mezcla del jarabe con el gas carbónico. Todos estos procesos son clave, ya que si falla uno de ellos, el refresco no tendrá buen sabor, el cual es uno de los requerimientos más importantes del cliente.

Es bien sabido por el personal que labora en las firmas refresqueras, que la mercadotecnia es vital para alcanzar el éxito, lo que se ha corroborado con la aplicación del QFD a la industria.

\section{REFERENCIAS}

Akao, Y. (1990). Quality function deployment: integrating customer requirements into product design. Productivity Press, Cambridge, Ma., 3-6.

ANPRAC. (2008). La industria de refrescos y aguas carbonatadas en 2008. 17.

Bottani, E. (2009). A fuzzy QFD approach to achieve agility. International Journal of Production Economics, DOI:10.1016/j.jpe.2009.02.013.

Carnevally, J. y Miguel, P. (2008). Review, analysis and classification of the literature on QFD - types of research, difficulties and benefits. International Journal of Production Economics, 114(2), 737-754.

Chan, L. y Wu, M. (2002). Quality function deployment: a literature review. European Journal of Operation Research, 143(3), 463-497.

Chang, K. y Chen, M. (2011). Applying the Kano model and QFD to explore customer's brand contacts in the hotel business: A study of a hot spring hotel. Total Quality Management, 22(1), 1-27.

Chaudhuri, A., y Bhattacharyyab. (2009). Acombined QFD and integer programming framework to determine attribute levels of conjoint study. International Journal of Production Research, 47(23), 6633-6649.

Chen, Ch., y Huang, S. (2011). Implementing KM programmes using fuzzy QFD. Total Quality Management, 22(4), 387-406.

Chen, Ch. y Shih, T. (2011). Implementing KM programmes using fuzzy QFD. Total Quality Management, 22(4), 387-406.

Chen, Y., Fung, R., y Tang, J. (2004). Fuzzy expected value modeling approach for determining target values of engineering characteristics in QFD. International Journal of Production Research, 43(17), 3583-3604. 
Delice, E., y Güngor, Z. (2011). A mixed integer goal programming model for discrete values of design requirements in QFD. International Journal of Production Research, 49(10), 2941-2957.

Excélsior. (05/09/2011).Méxicoes el mayorconsumidor de refresco en el mundo. Recuperado de http:// www.excelsior.com.mx/index.php?m=nota\&id_ nota $=766323$.

Golden, Bruce L., y Wasil, Edward A. (1987). Computerized vehicle routing in the soft drink industry. Operations Research, 35(1), 6-17.

Izar, J. M. (2011). Calidad y mejora continua: México: Lid.

Lee, A., Kang, H., Yang, Cheng, Y. y Chun, Y. (2010). An evaluation framework for product planning using FANP, QFD and multi-choice goal programming. International Journal of Production Research, 48(13), 3977-3997.

Milenio. (14/04/2012). Mexicanos gastan mil pesos al año en refrescos. Recuperado de http://www.zocalo. com.mx/seccion/articulo/mexicanos-gastan-milpesos-al-ano-en-refrescos.

Nasser, F., y Tabriz, I. (2009). Organizational support activities as facilitator in the QFD performance. International Journal of Management and Innovation, 1(2), 60-71.

Organización Editorial Mexicana (30/06/2008). Cada año, 152 litros de refresco por mexicano. Recuperado de http://www.oem.com.mx/laprensa/ notas/n752335.htm

Pullman, M., Moore, W., y Wardell, D. (2002). A comparison of quality function deployment and conjoint analysis in new product design. The Journal of Product Innovation and Management, 19(5), 354-365.

Shil, N., y Das, B. (2008). A study of customer satisfaction with regard to banking:An application of QFD. The Icfain Journal of Management Research, VII(8), 7-26.
Sullivan L.P. (1986). Quality function deployment. Quality Progress, 39-50.

Tontini, G. (2007). Integrating the Kano Model and QFD for designing new products. Total Quality Management, 18(6), 599-612.

Vinodh, S. y Suresh, C. (2011). Application of fuzzy QFD for enabling leanness in manufacturing organization. International Journal of Production Research, 49(6), 1627-1644.

24 Horas. (26/03/2012). Hacen menos amargo el precio de refrescos. Recuperado de http://www.24-horas. $\mathrm{mx} /$ hacen-menos-amargo-el-precio-de-refrescos/.
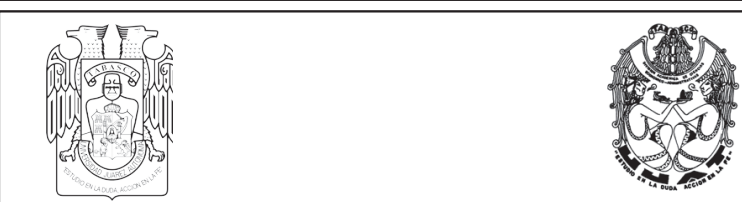

Universidad Juárez Autónoma de Tabasco División Académica de Ciencias

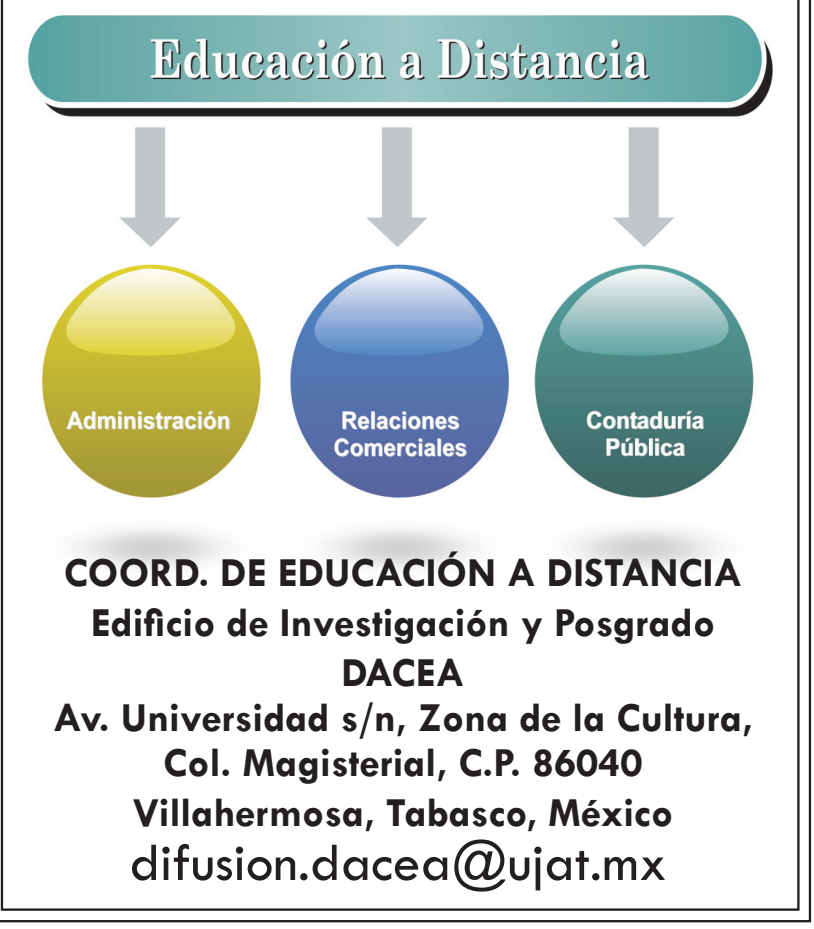

